\title{
SDN-enabled Platform for Energy-efficient Relay Protection Optical Port in Internet of Energy
}

\author{
Yanping Zhang ${ }^{1,}$, , Honghao Zhao ${ }^{2}$, Dan $\mathrm{Yi}^{1}$, Kai Liang ${ }^{1}$, Jun Liu ${ }^{1}$, Xiumin \\ Leng $^{1}$, Ying $\mathrm{CaO}^{3}, \mathrm{Xu}_{\text {Zhang }}{ }^{4}$ \\ ${ }^{1}$ State Grid Anshan Electric Power Supply Company, Anshan 114002, China \\ ${ }^{2}$ State Grid Liaoning Electric Power Company Limited, Shenyang 110006, China \\ ${ }^{3}$ Liaoning Planning and Designing Institute of Post and Telecommunication Company Limited, \\ Shenyang 110011, China \\ ${ }^{4}$ School of Computer Science and Engineering, Northeastern University, 110819, China \\ aemail: amengfb@163.com
}

Keywords: IoE, SDN, Energy transportation, Energy storage

\begin{abstract}
To improve the energy conversion ability and well utilize renewable resources via achieving a Peer-to-Peer (PtP) sharing mechanism in the form of bi-directional energy transportation, the concept of "Internet of Energy (IoE)" was put forward. Thus, in this paper, by virtue of Software-Defined Networking (SDN), a complementary platform is developed to make an effective decision for cooperative energy transmission and storage of relay protection optical port. The experiment results demonstrate the effectiveness of our solution.
\end{abstract}

\section{Introduction}

Our world has many types of potential energy generating ways that include electric power, petroleum, natural gas and renewable resources. However, due to the existence of industry barrier, the microgrids carrying diverse resources separately take their own load to satisfy the unique user demand, resulting in weak energy conversion ability and bad use of renewable resources. Moreover, the achievement of a Peer-to-Peer (PtP) energy sharing between Power Distribution Networks (PDNs) faces severe challenges because existing infrastructures lack the function of bi-directional (two-way) energy transportation. Also with imperfect energy storage facilities, it urgently requires a coordinative operation model.
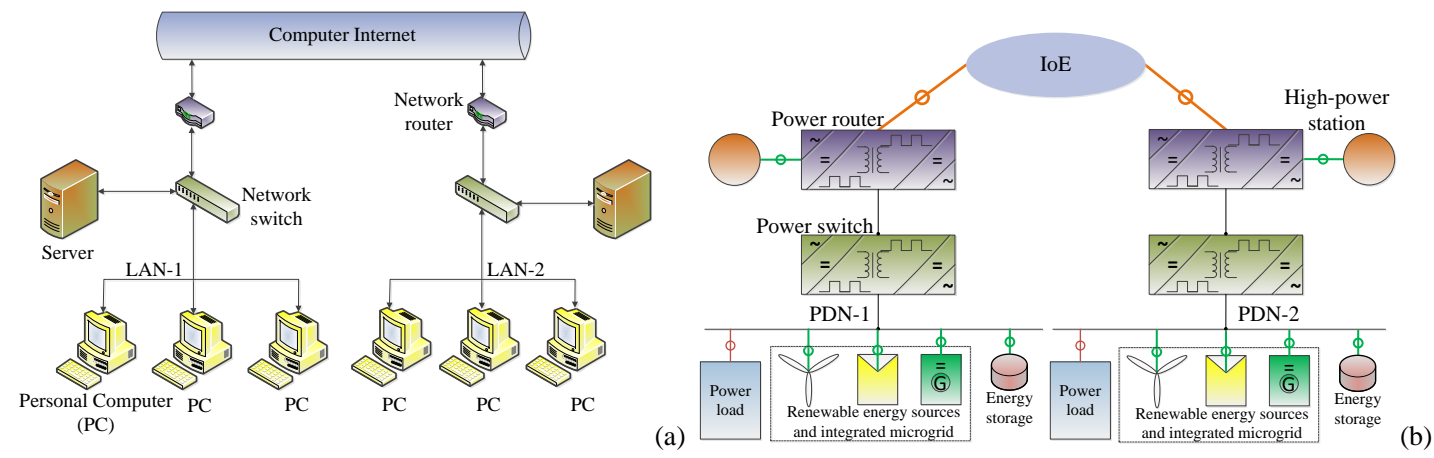

Fig. 1. Architecture similarity: a) computer Internet; b) Internet of Energy

To improve the energy conversion ability and well utilize renewable resources via achieving a PtP sharing mechanism in the form of bi-directional energy transportation, J. Rifkin firstly put forward the concept of "Internet of Energy (IoE)" in the recently published book "The third industrial revolution" [1]. Motivated by this concept, some famous research programs (e.g., FREEDM [2, 3] and E-energy [4]) were started. What's the meaning of IoE? To answer this question, we demonstrate the architecture similarity between computer Internet in Fig. 1(a) and a universal IoE in Fig. 1(b). In Fig. 1(a), Local Area Networks (LANs) are interconnected by network routers, while a network switch is responsible for fast data transferring among Personal Computers 
(PCs) within a single LAN [5-8]. Coincidentally, in Fig. 1(b), to support a PtP energy sharing between PDNs via bi-directional energy transportation, PDNs are interconnected by power routers (E-routers); the power switch achieves energy conversion between microgrids managed by a single PDN, and it supports dynamic renewable energy access while mitigating carbon emission. In addition, IoE has a strong ability of on-demand energy storage that makes active power supply and quality compensation.

Designing an effective cooperative energy transportation and storage mechanism is significantly meaningful as a key problem which has not been well solved in IoEs. Thus, in this paper, by virtue of Software-Defined Networking (SDN), a complementary platform is developed to make an effective decision for cooperative energy transmission and storage.

\section{Complementary SDN platform}

A new problem is where we make an effective decision for cooperative energy transmission and storage using aforementioned heuristics. In fact, it is inappropriate for power routers to make their own decision by complex self-learning in the existing IoE architecture, because the control collision has become very severe without utilizing a coordinated control. In addition, although some coordinated control approaches were proposed based on a multi-agent-based consensus algorithm, it is difficult for every power router to obtain the entire status information of all the others in a short time, so a real global smart control still cannot be achieved; moreover, these control applications solved only a single problem of voltage/frequency restoration or voltage synchronization.

SDN decouples the module of decision making and control from underlying switching devices, instead, a single centralized controller makes a decision according to gathered global network status information. This single centralized controller controls all underlying switching devices through distributing commands. The information collection and command distribution are both achieved in the manner of transferring flow tables over a secure channel (OpenFlow protocol) between the centralized controller and underlying switching devices. A list of problems solved by smart control can be seen as APPs (applications) connected to the northbound interface of the centralized controller [5.

For this end, we develop a complementary SDN-enabled platform of making the most effective decision for cooperative energy transportation and storage using aforementioned heuristics. As an instance of Fig. 2(a), the power router supporting OpenFlow protocol has only one function of energy transportation and storage, and it notifies the status information encapsulated in flow tables to the centralized controller. Thus, as shown in Fig. 2(b), the rule field of a flow table will record the status information of energy generating, power load and energy storage owned by the PDN connected to the corresponding power router; next, the centralized controller makes a decision that includes the selection of transportation ports at relative power routers, according to heuristic results obtained by gathering status information and APP requirement in Fig. 2(a). Thus, in Fig. 2(b), the rule field will contain the flow entry of I/O port, while the action filed will include the operation of establishing/removing energy transportation paths; the statistics field will be responsible for computing the energy transportation cost once the corresponding path has been determined.

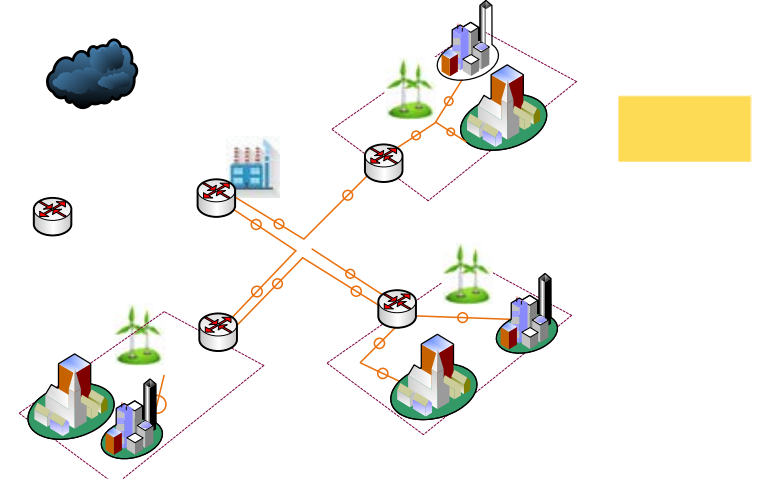

Fig. 2. Complementary SDN platform 


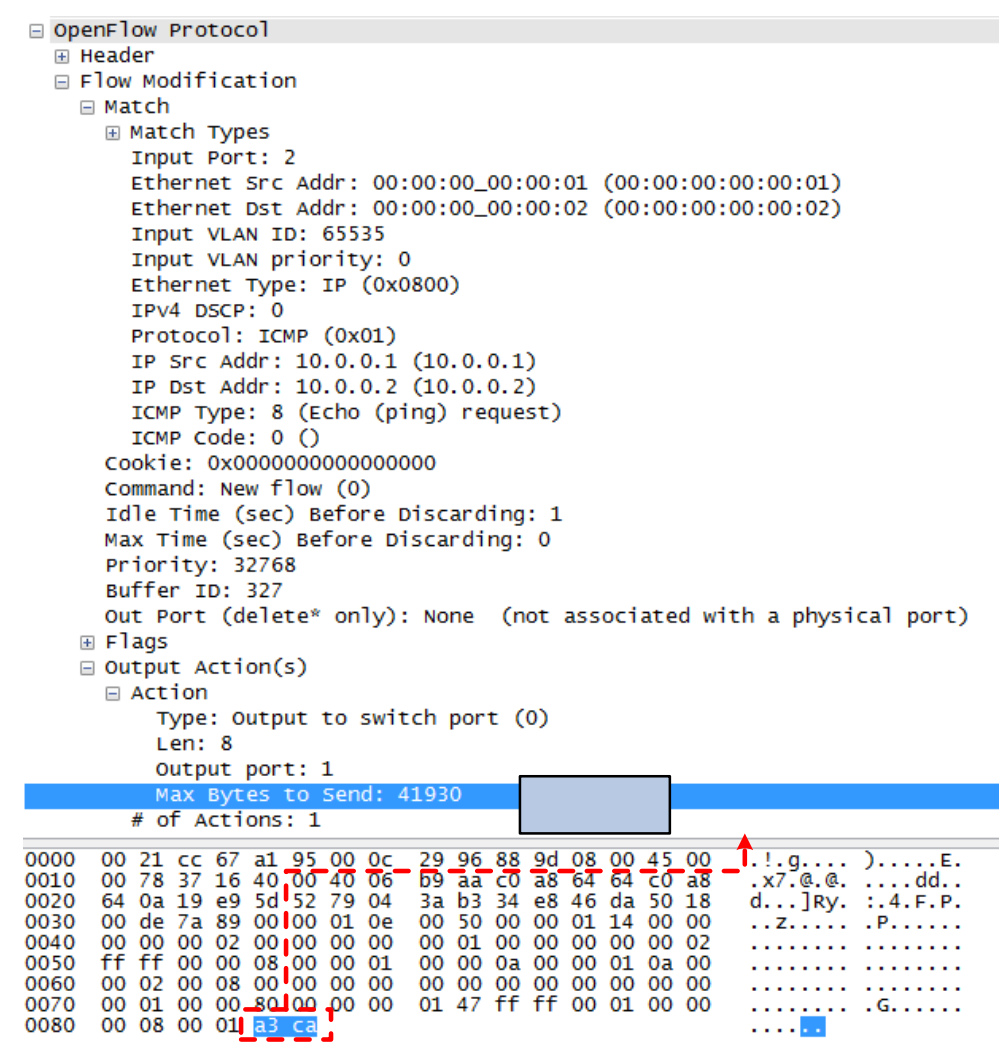

Fig. 3. Wireshark capture for energy transportation and storage function

\section{Experiment results and discussions}

We make the following expansion of Flow Mod messages for OpenFlow protocol: 1) add time-variable and energy-related information of energy generating, power load and energy storage in Fig. 3; 2) add I/O port information of the current power router traversed by an energy transportation path so that we can compute the corresponding transportation cost.

In, Fig. 4, we also evaluate the processing latency of the centralized controller taking the aforementioned expansion of flow tables into account. The processing latency of the centralized controller here is the time duration from receiving one power packet to distributing Flow Mod message, and we test this parameter using 10 different groups of power packets. The experimental result shows that we achieve a cooperative energy transportation and storage function with a slight additional processing latency in a microsecond (us) level.

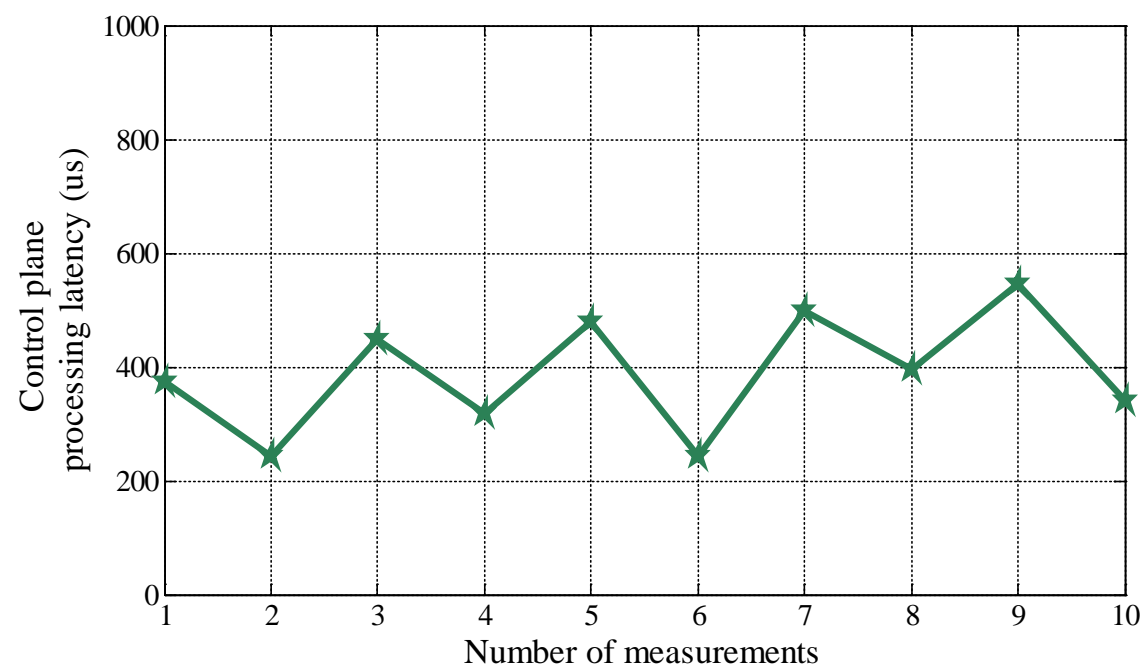

Fig. 4. Experiment result of control plane processing latency 


\section{Conclusion}

In this paper, we have proposed a novel SDN-enabled platform for energy transportation and storage in IoEs. The feasibility of establishing a complementary SDN platform has been experimentally demonstrated via the Wireshark capture of relative flow table, and our platform has an acceptable microsecond processing latency of the control plane.

\section{References}

[1] J. Rifkin, The third industrial revolution: how lateral power is transforming energy, the economy, and the world, New York: Palgrave Macmillan, pp. 31-46, 2013.

[2] L. M. Crow, B. McMillin, W. Wang, S. Bhattacharyya, Intelligent energy management of the FREEDM system, in Proc. of IEEE PES General Meeting, pp. 1-4, 2010.

[3] Q. A. Huang, L. M. Crow, T. G. Heydt, P. J. Zheng, J. S. Dale, The future renewable electric energy delivery and management (FREEDM) system: the energy Internet, Proceedings of the IEEE, vol. 99, no. 1, pp. 133-148, 2011.

[4] European commission, Towards smart power networks, lessons learned from European research FP5 projects, Raooirt EUR 21970, 2005.

[5] W. Hou; L. Guo; X. Wei; Y. Liu; Q. Song. Virtual network planning for converged optical network and data centers: Ideas and challenges, IEEE Network, 27(6), pp. 52-58, 2013.

[6] W. Hou; R. Zhang; W. Qi, K. Lu; J. Wang; L. Guo. A provident resource defragmentation framework for mobile cloud computing, IEEE Transactions on Emerging Topics in Computing, PP(99), pp. 1-12, 2015.

[7] W. Hou; L.Guo; Y. Liu, C. Yu, Y. Zong. Resource management and control in converged optical data center networks: survey and enabling technologies, Computer Networks, 88(2015): 121-135, 2015.

[8] W. Hou, C. Yu, Y. Zong. A novel dynamic virtual infrastructure planning for converged optical network and data centers under power outage and evolving recovery, Optical Switching and Networking, 14, pp. 209-216, 2014. 